\title{
Insufficient evidence of the effect of systemic antibiotics on adults with symptomatic apical periodontitis or acute apical abscess
}

\author{
Abstracted from \\ Cope A, Francis N, Wood F, Mann MK, Chestnutt IG.

\section{Question: Are systemic antibiotics provided with or without surgical intervention, with or without analgesics effective in treating symptomatic apical periodontitis or acute apical abscess in adults?}

Systemic antibiotics for symptomatic apical periodontitis and acute apical abscess in adults. Cochrane Database Syst Rev 2014; 6: Art. No. CD010136. DOI: 10.1002/14651858.CD010136.pub2.

Address for correspondence: Luisa Fernandez Mauleffinch, Review Group Co-ordinator, Cochrane Oral Health Group, School of Dentistry, The University of Manchester, Coupland III Building, Oxford Road, Manchester, M13 9PL, UK. E-mail: luisa.fernandez@manchester.ac.uk

Data sources The Cochrane Oral Health Group's Trials Register, Cochrane Central Register of Controlled Trials, Medline, Embase, CINAHL, OpenGrey, ZETOC Conference Proceedings World Health Organization (WHO) International Trials Registry Platform and the US National Institutes of Health Trials Registry databases were searched with no restrictions on the language or date of publication.

Study selection Randomised controlled trials of systemic antibiotics in adults with a clinical diagnosis of symptomatic apical periodontitis or acute apical abscess, with or without surgical intervention (considered in this situation to be extraction, incision and drainage or endodontic treatment) and with or without analgesics.

Data extraction and synthesis Study selection, data abstraction and risk of bias assessment were carried out independently by two reviewers.

Results Two trials involving 62 patients were included. They compared the effects of oral penicillin $V$ potassium versus a matched placebo given in conjunction with a surgical intervention and analgesics to adults with an acute apical abscess or symptomatic necrotic tooth. One study was considered to have a high risk of bias and the other an unclear risk of bias. The primary outcomes were patient-reported pain and swelling. There were no statistically significant differences in participant-reported measures of pain or swelling at any of the time points assessed within the review. The body of evidence was assessed as at very low quality. Conclusions There is very low quality evidence that is insufficient to determine the effects of systemic antibiotics on adults with symptomatic apical periodontitis or acute apical abscess.

This paper is based on a Cochrane Review published in the Cochrane Library 2014, issue 6 (see www.thecochranelibrary.com for information). Cochrane Reviews are regularly updated as new evidence emerges and in response to feedback, and the Cochrane Library should be consulted for the most recent version of the review.

\section{Commentary}

Antibiotic resistance and the indiscriminate use of prescription antibiotics is a worldwide problem. ${ }^{1}$

It is known that the inappropriate use of antibiotics is one of the main causes of antibiotic resistance. In medicine the problem increases since it is associated with the increase in mortality of patients in hospitals due to antibiotic resistant pathology. ${ }^{2}$

Health providers are to blame when the prescriptions are not necessary. In spite of this, patients need to be informed of the consequences of misuse of antibiotics in conditions where no bacteria are associated with the illness.

A recent systematic review concluded that there is a lack of knowledge in the general population about antibiotics and there is a need to strengthen educational initiatives for patients, and for health care providers to inform patients about antibiotic consumption. ${ }^{3}$

In dentistry the use of antibiotics still needs to be explored such as in endodontics, where apical periodontitis and acute apical abscesses are associated with bacteria colonising the dental canal.

The review had two objectives, the addition of systemic antibiotics with or without surgical intervention for the treatment of symptomatic apical periodontitis or acute apical abscess. The dental conditions are associated with pain.

The Cochrane systematic review after a thorough search in English databases included two studies in the final selection. The two studies used penicillin $\mathrm{V}$ compared to placebo in the surgical treatment of symptomatic apical periodontitis or acute apical abscess, in both trials patients received analgesics. No trials were found for the second objective; the use of antibiotics versus placebo without a surgical intervention.

The grade of the evidence assessed by the authors was very low for the outcomes of pain and swelling at 24/48 and $72 \mathrm{hrs}$ and concluded that there is insufficient evidence to determine the effects of antibiotics on adults with symptomatic apical periodontitis or acute apical abscess. One must take into account that everyone's pain threshold is different thereby making the outcome subjective with an introduction of bias. From a clinical standpoint, it is absolutely critical that a thorough history is taken that will help determine the length of time the patient has been in pain and if there have been any systemic manifestations of infection such as a fever. In addition to radiographic analysis of periapical pathology, clinical evidence of intraoral and/or extraoral swelling should be noted. It is uncertain and more trials are suggested to guide practitioners in the management of acute dental infections. Practitioners should weigh the risk and benefits of the 
prescription of systemic antibiotics and diagnose appropriately the patient's present condition.

Prescriptions of systemic antibiotics are still necessary but it is important to instruct patients about the use of antibiotics and the possible consequences of misuse.

Analia Veitz-Keenan and Angela M De Bartolo

NYU College of Dentistry, New York, USA
1. WHO. The evolving threat of antimicrobial resistance. Options for action. http:// whqlibdoc.who.int/publications/2012/9789241503181_eng.pdf [Accessed $9^{\text {th }}$ November 2014]

2. CDC. Antibiotic/Antimicrobial Resistance. Threat Report 2013. http://www.cdc.gov/ drugresistance/threat-report-2013 [Accessed 23 November 2014]

3. Gualano M, Gili R, Scaioli G, Bert F, Siliquini R. General population's knowledge and attitudes about antibiotics: a systematic review and meta-analysis. Pharmacoepdemiol Drug Saf. 2014; Sep 24. doi: 10.1002/pds.3716. [Epub ahead of print]

Evidence-Based Dentistry (2014) 15, 104-105. doi:10.1038/sj.ebd.6401058 